Rev. salud pública. 8 (1): 108-117, 2006

Ensayo/Essay

\title{
Contaminación por Monóxido de Carbono: un Problema de Salud Ambiental
}

\author{
Jairo Téllez ${ }^{\mathrm{I}}$, Alba Rodríguez ${ }^{\mathrm{II}}$ y Álvaro Fajardo ${ }^{\mathrm{III}}$ \\ I Médico. M. Sc. Toxicología. Especialista Salud Ocupacional. Especialista Farmacodependencia. \\ Departamento de Toxicología, Facultad de Medicina, Universidad Nacional de Colombia. E-mail: \\ jatellezm@unal.edu.co \\ II Médica. M. Sc. Toxicología. Especialista Salud Ocupacional. Departamento de Toxicología, Facultad \\ de Medicina, Universidad Nacional de Colombia. E-mail: airodriguezp@unal.edu.co \\ III Médico. Ingeniero Industrial. Especialista en administración de Salud Ocupacional. Departamento de \\ Toxicología, Facultad de Medicina, Universidad Nacional de Colombia. E-mail: alfajardoz@unal.edu.co
}

Recibido 29 Junio 2005/Enviado para Modificación 31 Diciembre 2005/Aceptado 9 Febrero 2006

\section{RESUMEN}

El monóxido de carbono es considerado uno de los mayores contaminantes de la atmósfera terrestre. Sus principales fuentes productoras responsables de aproximadamente $80 \%$ de las emisiones, son los vehículos automotores que utilizan como combustible gasolina o diesel y los procesos industriales que utilizan compuestos del carbono. Esta sustancia es bien conocida por su toxicidad para el ser humano. Sus efectos tóxicos agudos incluida la muerte han sido estudiados ampliamente; sin embargo, sus potenciales efectos adversos a largo plazo son poco conocidos. En los últimos años, los estudios de investigación experimentales en animales y epidemiológicos en humanos han evidenciado relación entre población expuesta en forma crónica a niveles medios y bajos de monóxido de carbono en aire respirable y la aparición de efectos adversos en la salud humana especialmente en órganos de alto consumo de oxígeno como cerebro y corazón. Se han documentado efectos nocivos cardiovasculares y neuropsicológicos en presencia de concentraciones de monóxido de carbono en aire inferiores a 25 partes por millón y a niveles de carboxihemoglobina en sangre inferiores a $10 \%$. Las alteraciones cardiovasculares que se han descrito son hipertensión arterial, aparición de arritmias y signos electrocardiográficos de isquemia. Déficit en memoria, atención, concentración y alteraciones del movimiento tipo parkinsonismo, son los cambios neuropsicológicos con mayor frecuencia asociados a exposición crónica a bajos niveles de monóxido de carbono y carboxihemoglobina. 
Palabras Clave: Monóxido de carbono, carboxihemoglobina, salud ambiental, salud pública, toxicología, marcadores biológicos (fuente: DeCS, BIREME)

\begin{abstract}
Carbon monoxide contamination: an environmental health problem

Carbon monoxide is considered to be a major factor contaminating earth's atmosphere. The main sources producing this contamination are cars using gasoline or diesel fuel and industrial processes using carbon compounds; these two are responsible for $80 \%$ of carbon monoxide being emitted to the atmosphere. This substance has a well-known toxic effect on human beings and its acute poisonous effects (including death) have been widely studied; however, its long-term chronic effects are still not known. During the last few years, experimental research on animals and studies of human epidemiology have established the relationship between chronic exposure to low and middle levels of carbon monoxide in breathable air and adverse effects on human health, especially on organs consuming large amounts of oxygen such as the heart and brain. Harmful cardiovascular and neuropsychological effects have been documented in carbon monoxide concentration in air of less than $25 \mathrm{ppm}$ and in carboxyhaemoglobin levels in blood of less than $10 \%$. The main cardiac damage described to date has been high blood pressure, cardiac arrhythm and electrocardiograph signs of ischemia. Lack of memory, attention, concentration and Parkinson-type altered movement are the neuropsychological changes most frequently associated with chronic exposure to low levels of carbon monoxide and carboxyhaemoglobin.
\end{abstract}

Key Words: Carbon monoxide, carboxyhemoglobin, environmental health, public health, toxicology, biologic markers (source: $M e S H, N L M$ ).

$\mathrm{E}$

1 monóxido de carbono es un gas incoloro e inodoro que se forma por la combustión incompleta de material orgánico, en presencia deficitaria de oxígeno. Es considerado uno de los mayores contaminantes de la atmósfera terrestre, y uno de los mayores problemas ambientales de América Latina $(1,2)$. Las principales fuentes productoras de este contaminante son los vehículos automotores que utilizan como combustible gasolina o diesel; los procesos industriales; los incendios forestales y urbanos y la incineración de materia orgánica. Los vehículos automotores y los procesos industriales son responsables de aproximadamente $80 \%$ de las emisiones de monóxido de carbono a la atmósfera $(1,3)$.

Con el uso creciente de electrodomésticos y gasodomésticos, además de otros elementos como chimeneas y sistemas de calefacción, las viviendas se 
han tornado en un factor de riesgo importante de intoxicación intradomiciliaria por monóxido de carbono (4).

De acuerdo con la Environmental Protection Agency, en los Estados Unidos las emisiones de monóxido de carbono al ambiente son producidas en un $95 \%$ por los vehículos automotores (5). La Comisión Nacional del Medio Ambiente de Chile informó que para el año 2000, el 91 \% de las emisiones de monóxido de carbono en la región metropolitana de Santiago de Chile fueron producidas por el transporte automotor (6).

En México, los vehículos automotores también son responsables de la mayor cantidad de emisiones de monóxido de carbono a la atmósfera; allí se ha fijado como concentración media de este compuesto para 1 hora el valor de 11 partes por millón, que equivale a 100 puntos del Índice Metropolitano de Calidad del Aire (7).

En Brasil, el monóxido de carbono es el polutante emitido en mayor cantidad a la atmósfera. En Sao Paulo 1,5 millones de toneladas son lanzadas al aire anualmente; de estos el $78 \%$ son producidos por los automotores a gasolina o diesel, $15 \%$ por automotores con combustible alcohol, $3 \%$ por motocicletas, $2 \%$ por taxis y $2 \%$ resultan de procesos industriales (8).

En Colombia, el Instituto de Hidrología, Meteorología y Estudios Ambientales, estimó para 1996 las emisiones atmosféricas de gases con efecto local en 8612 kilotoneladas, de las cuales el monóxido de carbono representó el 58 \% (9). En Bogotá, el Departamento Administrativo del Medio Ambiente estima que la concentración media de monóxido de carbono atmosférico en un día normal se encuentra entre 30 y 35 partes por millón. En el informe sobre monitoreo realizado en el “día sin carro” en 2005, se estimó que la concentración ambiental de monóxido de carbono disminuyó en un 76 $\%$ respecto a un día normal (10).

\section{EFECTOS TÓXICOS EN LA SALUD HUMANA}

Para 1999 la Asociación Americana de Centros para el Control de Intoxicaciones y el Sistema de Vigilancia de Exposición a Tóxicos, informaron sobre 17006 casos de intoxicación y 35 muertes relacionados con exposición a monóxido de carbono. Omaye, informa que anualmente en Estados Unidos entre 10000 a 40000 personas demandan atención médica o faltan al trabajo debido a intoxicación por monóxido de carbono (11). 
Gómez y Valcarce en trabajo realizado sobre 882 personas fallecidas en el periodo de 1991 a 2000, encontraron que los periodos del año que con mayor frecuencia se presentaron casos relacionados con monóxido de carbono, son los correspondientes a otoño e invierno, en una estrecha relación con el uso de calderas, calefacciones, braseros y chimeneas en los hogares (4). En 1986, Kosada, reportó en España 31 casos de intoxicación por monóxido de carbono; Portoles y Algarra informaron en 1992 una serie de 13 casos de intoxicación aguda por monóxido de carbono en España (12). Revert y Brotons describieron en 1995 una epidemia de intoxicación aguda por monóxido de carbono en la Región de Badía, relacionada con escapes en equipos de calefacción en temporada de invierno. Dueñas, Ruiz-Mambrilla y otros en 1997 informaron sobre 154 casos de intoxicación por monóxido de carbono presentados en 2 hospitales de la región de Valladolid en España en un periodo de 18 meses; de estos casos 5 fueron mortales (13).

El monóxido de carbono, que por sus características fisico-químicas es llamado "el asesino silencioso", utiliza múltiples mecanismos de toxicidad que explican sus potenciales efectos adversos en la salud humana. Entre ellos se encuentran los siguientes: Compite con el oxigeno y altera la curva de disociación de la hemoglobina. Una vez penetra al organismo, el monóxido de carbono se une a las enzimas del grupo Hem de la hemoglobina, desplazando al oxigeno de la misma. De esta manera se forma en la sangre un complejo que se denomina carboxihemoglobina, que dificulta el transporte de oxigeno a las células y tejidos, lo que va a producir una hipoxia celular generalizada. La molécula de hemoglobina dispone de cuatro sitios de unión con el oxígeno, cuando uno de estos sitios es ocupado por el monóxido de carbono, la hemoglobina se altera de tal forma que impide que los otros sitios se unan al oxigeno, produciendo así una desviación de la curva de disociación oxígeno-hemoglobina hacia la izquierda. Esta alteración empeora la hipoxia celular producida por la formación de carboxihemoglobina. Los síntomas y signos iniciales de la intoxicación aguda se presentan muy rápidamente y se han relacionado con niveles de carboxihemoglobina en sangre superiores a $10 \%(12,14-18)$.

Interfiere la utilización del oxigeno por los tejidos El carácter fuertemente ligante del monóxido de carbono le permite unirse a los átomos de hierro de los citocromos, especialmente a los que intervienen en la cadena respiratoria mitocondrial, lo que impide una adecuada utilización del oxigeno por los tejidos orgánicos, agravando la hipoxia tisular originada en el déficit de oxígeno en la hemoglobina $(15,16)$.

Produce peroxidación lipídica cerebral 
Se cree que el monóxido de carbono reacciona con la enzima xantína deshidrogenasa convirtiéndola en xantina oxidasa, la cual a su vez reacciona con la hipoxantina, produciendo superóxidos; estos reaccionan con el óxido nítrico producido por el endotelio formando peroxinitrito, ácido peroxinitroso y/o peroxinitratos, potentes oxidantes que interactúan con los ácidos grasos insaturados, produciendo peroxidación lipídica y originando desmielinización progresiva de las neuronas del sistema nervioso central $(16,19,20)$. Sherral Devine y otros presentan un caso clínico en el cual una mujer que laboró durante un largo periodo de tiempo como cocinera en un restaurante, presentó lesiones neuropsicológicas caracterizadas por dificultades en la lectura, la escritura y en el lenguaje verbal; estas alteraciones fueron correlacionadas con múltiples lesiones bilaterales en los ganglios basales detectadas mediante Resonancia Nuclear Magnética. Estos autores sugieren que la neurotoxicidad por monóxido de carbono, también involucra otros mecanismos de toxicidad como la inhibición de la respiración mitocondrial, acidosis metabólica y peroxidación lipídica cerebral, lo que conlleva a una desmielinización progresiva de la sustancia blanca cerebral (20).

Inhibe la síntesis aeróbica de adenosina trifosfato

La alteración en el transporte de electrones en la mitocondria produce estrés oxidativo, que se manifiesta por un aumento en la fracción de radicales libres hidroxilo $(14,21)$.

Formación de grupos sulfatides en cerebro La actividad de la enzima arilsulfatasa A es esencial en el metabolismo de la mielina, ya que participa en su degradación normal e impide la acumulación de grupos sulfatides que son neurotóxicos. Cuando la actividad de la enzima arilsulfatasa A es inferior al $10 \%$, se produce acumulación de los grupos sulfatides, generándose una leucoencefalopatía. Individuos que presenten una disminución previa de la actividad de esta enzima en porcentajes entre $10 \%$ y $30 \%$ que no se ha expresado clínicamente, al exponerse a monóxido de carbono, desarrollan un cuadro clínico de encefalopatía retardada (22). La encefalopatía retardada por monóxido de carbono es un cuadro clínico poco conocido y de rara presentación; se describe que tiene una frecuencia de 2,7 $\%$, sus características clínicas más frecuentes son deterioro cognitivo, alteraciones de la marcha, incontinencia de esfínteres, mutismo, hiponimia, hipertonía y retropulsión. Este cuadro se suele presentar en mayores de 40 años de edad, su patogénesis se ha relacionado con disminución de la actividad de la enzima arilsulfatasa A, por debajo del $10 \%$, lo que permite la acumulación de grupos sulfatides, que son considerados neurotóxicos al generar un cuadro de leucoencefalopatía $(22,23)$. 
En intoxicación crónica las manifestaciones clínicas pueden variar dependiendo del tiempo de exposición, las concentraciones del tóxico y la susceptibilidad individual. Los principales efectos crónicos por exposición a monóxido de carbono son alteraciones cardiovasculares y neuropsicológicas.

Las alteraciones cardiovasculares se caracterizan por incremento en los niveles de lípidos plasmáticos, facilita la adhesión y agregación plaquetaria, lo que favorece el proceso arterioesclerótico y aumenta la probabilidad de desarrollar hipertensión arterial sistémica y episodios de trombosis $(24,25)$. Arritmias supraventriculares y ventriculares, Supradesnivel o infradesnivel del segmento S-T, prolongación del intervalo Q-T e inversión de la onda T son las alteraciones de la actividad electrocardiográfica más frecuentemente reportadas. Estas alteraciones se han descrito incluso en individuos con niveles de carboxihemoglobina en sangre inferiores a $6 \%(14,26)$.

Gandini y Castoldi reportan un caso clínico en un niño de 12 años expuesto a monóxido de carbono por defectos en el sistema de calefacción de su hogar, que se encontraba asintomático y presentaba niveles bajos de carboxihemoglobina, le fueron encontrados signos de isquemia miocárdica en el electrocardiograma; el autor señala la necesidad de tener presente que con niveles bajos de carboxihemoglobina pueden presentarse alteraciones cardiacas (27). Koskela y Mutanen, en estudio de seguimiento por un periodo de 14 años en trabajadores de fundición expuestos a monóxido de carbono, estimaron el valor predictivo del examen médico para enfermedad cardiovascular y muerte. Los resultados mostraron mayor incidencia de hipertensión arterial y de enfermedad coronaria en expuestos que en no expuestos; en el electrocardiograma encontraron presencia de onda Q, extrasístoles ventriculares y alteraciones en el segmento S-T (28).

Las alteraciones neuropsicológicas se relacionan con presencia de cefalea persistente, fatiga, reducción de la percepción visual, la destreza manual, disminución de memoria, disminución de la concentración, atención y las capacidades para conducir, trastornos del sueño con predominio de insomnio, irritabilidad y con menos frecuencia deterioro demencial. También se ha relacionado la exposición crónica a monóxido de carbono con la aparición de un cuadro neurológico similar al parkinson. $(12,15,29,30)$.

Venegas presenta el caso de una mujer de 43 años de edad, que fue encontrada en su apartamento en estado de inconciencia; esta mujer tenia antecedente de exposición a monóxido de carbono. Dos semanas después de salir del hospital inició deterioro cognitivo progresivo, alteraciones de la marcha y pérdida del control de esfínteres; bradicinesia generalizada, tendencia a 
la retropulsión espontánea y exaltación de los reflejos de prensión, búsqueda y succión. La tomografía axial computarizada de cerebro mostró hipodensidad de ambos globos pálidos; una resonancia magnética reveló extensa lesión desmielinizante de sustancia blanca hemisférica y lesión lacunar del globo pálido derecho. Cuatro meses después de iniciado el cuadro clínico, presentó recuperación; se realizó un diagnóstico de encefalopatía retardada por monóxido de carbono (23). Sohn describe un caso en un hombre de 56 años de edad hospitalizado con signos de parkinsonismo y deterioro de memoria un mes después de un episodio de intoxicación por monóxido de carbono. La resonancia magnética funcional mostró reducción del transportador de dopamina y daño de la sustancia blanca. Dos meses después de iniciado su cuadro clínico presentó mejoría de sus signos de parkinsonismo, pero sus problemas de memoria persistieron durante 2 meses más (22).

\section{MONITOREO DE LA EXPOSICIÓN A MONÓXIDO DE CARBONO}

El monitoreo de la exposición a monóxido de carbono, se puede realizar a través de la determinación de biomarcadores y medición de concentraciones ambientales. El término biomarcador es usado para incluir casi cualquier medida que refleje una interacción entre un sistema biológico y un riesgo potencial. Los biomarcadores también han sido definidos como cambios inducidos por un contaminante en los componentes bioquímicos o fisiológicos que pueden ser medidos en un sistema biológico $(31,32)$.

Carboxihemoglobina en sangre. Es un biomarcador de exposición de alta especificidad, refleja la dosis interna de monóxido de carbono en sangre. También se considera un biomarcador de efecto precoz en cuanto refleja alteraciones bioquímicas y fisiológicas en la estructura de la hemoglobina y en el proceso fisiológico de oxigenación celular y tisular. La American Conference Governmental Industrial Hygienist, fija el valor biológico tolerable para carboxihemoglobina en sangre en 3,5 \% (33).

Actividad de la enzima arilsulfatasa A. Este es un biomarcador de susceptibilidad. La enzima arilsulfatasa A es esencial en el metabolismo de la mielina, ya que participa en su degradación normal e impide la acumulación de grupos sulfatides. Cuando la actividad de la enzima arilsulfatasa A es inferior al $10 \%$, se produce acumulación de los grupos sulfatides. Individuos que presenten una disminución previa de la actividad de esta enzima en porcentajes entre $10 \%$ y $30 \%$ que no se ha expresado clínicamente, al exponerse a monóxido de carbono, desarrollan un cuadro clínico de encefalopatía retardada $(22,23)$. 
Monóxido de carbono exhalado. Marcador de exposición de alta especificidad, que permite tener una medición de dosis interna. La American Conference Governmental Industrial Hygienist, fija su valor límite biológico en 20 partes por millón (33).

Monitoreo ambiental

- TLV-TWA: El TWA o concentración máxima en aire aceptada para 8 horas diarias de trabajo con máximo 40 horas semanales de exposición a monóxido de carbono, el adoptado por la American Conference Governmental Industrial Hygienist es de 25 partes por millón (33).

- Ambiente General: El estándar de calidad de aire para monóxido de carbono fijado por la Organización Mundial de la Salud es de 35 partes por millón por hora (2) •

\section{REFERENCIAS}

1. Davies R, Buss DC, Routledge PA. Monóxido de Carbono. En: Manual de Toxicología Básica. Mencías E, Mayero LM. Madrid: Editorial Díaz de Santos; 2000. pp 593-595.

2. Organización Panamericana de la Salud [Internet]. Contaminación ambiental. Disponible en: http: www.paho.org. Consultado Enero 2005.

3. Córdoba D, Ramos JI. Monóxido de Carbono. En: Toxicología. Cordoba D. $4^{\circ}$ edición. Bogotá: Editorial el Manual Moderno; 2001. pp. 313-315.

4. Gómez J, Valcarce F. Tóxicos detectados en muertes relacionadas con fuegos e intoxicaciones por monóxido de carbono. Rev. Toxicol. 2003; 20:38-42.

5. U.S Environmental Protection Agency. National air quality 2001 status and trends. Carbon Monoxide; 2001.

6. Consejo Nacional del Medio Ambiente de Chile [Internet]. Disponible en: htpp: www.conama.cl. Consultado Enero 2005.

7. Sistema de Información del Medio Ambiente de México [Internet]. Índice metropolitano de calidad del aire. Disponible en: http: www.sima.com.mex Consultado Enero 2005.

8. Universidade Federal de Santa Catarina [Internet]. Caderno digital de informaçao sobre energía, ambiente e desenvolvimiento. Disponible en: http:

www.guiafloripa.com.br. Consultado Enero 2005

9. Chaparro LR, Cuervo MP, Gómez J, Toro MA. Emisiones al ambiente en Colombia. En: El medio ambiente en Colombia. Colombia: Instituto de Hidrologìa, Meteorología y Estudios Ambientales-IDEAM;2001. pp. 530542.

10. Departamento Administrativo del Medio Ambiente-DAMA [Internet]. Informe del día sin carro en Bogotá. Disponible en: http: www. Dama.gov.co Consultado Enero de 2005. 
11. Omaye ST Metabolic modulation of carbon monoxide toxicity. Toxicology 2002; 180:139-150.

12. Portoles A, Algarra J, Tarquis P, Vargas E, Jiménez de Diego L. Intoxicación por monóxido de carbono a propósito de 13 casos. Rev. Clin. Esp. 1982; 191:317-319.

13. Dueñas A, Ruiz-Mambrilla M, Gandía F, Cerda R, Escudero JC, Pérez J, Díaz G. Epidemiology of acute carbon monoxide poisoning in a Spanish Region. Clinical Toxicology 2001; 39(1):53-57.

14. Raud JA, Mathieu-Wolf M, Hampson NB, Thom SR. Carbon monoxide poisoning a public health perspective. Toxicology 2000; 145(1):1-14.

15. Ladrón de Guevara J, Moya V. Carbono y sus compuestos inorgánicos. En: Toxicología Médica y Laboral. Ed. Panamericana. McGraw-Hill; 1995 pp. 196-204.

16. Piantadosi CA. Carbon monoxide poisoning. New England Journal Medicine 2002; 347(14):1054-1055.

17. Crespo JM, Sesar A, Misa MJ, Requena I, Arias M. Pseudomigraña como manifestación de intoxicación por monóxido de carbono. Rev Neurol. 2001; 32 (11): 1047.

18. Ares B, Casais JL, Dapena D, Lema M, Prieto JM. Cefalea secundaria a intoxicación por monóxido de carbono. Rev Neurol. 2001; 32 (4): 339-341.

19. Amitai Y, Zlotogorski Z, Golan-Katsav V, Wexler A, Gross D. Neuropsychological impairmeint from acute low-level exposure to carbon monoxide. Archives neurol. 1998; 55(6): 845-848.

20. Sherral D, Shalene K, Carole P and Roberta W. MRI and neuropsychological correlates of carbon monoxide exposure: a case report. Environmental Health Perspectives, 2002, 110(10): 1051-1055

21. Miro O, Cardellach F, Alonso JR, Casademont J. Physiopathology of acute carbon monoxide poisoning. Med. Clin. 2000, 114(17):678

22. Sohn Y, Jeong Y, Kim H, Kim JS. The brain lesion responsible for parkinsonism after carbon monoxide poisoning. Arch Neurol. 2000; 57: 12141218.

23. Venegas-Franeke P, Miranda M, Delgado C. Encefalopatía retardada por monóxido de carbono. Rev Neurol. 2001; 33 (10): 996-997.

24. Barbe C, Rochetaing A, Creer P, Cardiovascular effects subcronically low/high carbon monoxide exposure in rats. Environmental Toxicology and Pharmacology 1999; 8:23-31.

25. Martín JC, Boubert P, Zerbib D, Conso F. [Internet]. Intoxication par l'oxyde de carbone d'origine professionnelle. Disponible en : htpp:www.univ-paris. Consultado Abril 2005.

26. Gandini C, Castoldi A, Candura S, Locatelli C, Butera R, Priori S, Manzo L. Carbon monoxide cardiotoxicity. Clinical Toxicology 2001; 39(1): 35-44.

27. Gandini C, Castoldi A, Candura S, Priori S, Locatelli C, Butera R, Ballet C, Manzo L. Cardiac damage in pediatric carbon monoxide poisoning. Clinical Toxicology 2001; 39(1): 45-51. 
28. Koskela R-S, Mutanen P, Sorsa J-A, Klockars M. Factor predictive of ischemic heart disease mortality in foundry workers exposed to carbon monoxide. American Journal of Epidemiology 2000; 152(7): 628-632.

29. Environmental Protection Agency. USA. Carbon monoxide and nervous system; 2004.

30. Raud JA, and Benignus VA. Carbon monoxide and the nervous system. Neuroscience and Biobehavioral Reviews 2002; 26(8):925-940.

31. McCunney R. Clinical applications of biomarkers in Occupational Medicine. In: Biomarkers and Occupational Health: progress and perspectives; United States: The National Academy of Sciences;1995.

32. World Health Organization. Environmental Health Criteria 22. Biomarkers and Risk assessment: concepts and principles. Geneva;1993.

33. American Conference Governmental Industrial Hygienist. TLVs and BEIs. Cincinnati, Ohio; 2002. 